\title{
Chaos, potential predictability and model validation of climate variations
}

\author{
C. S. Frederiksen* $\quad$ X. Zheng ${ }^{\dagger}$
}

(Received 7 August 2000)

\begin{abstract}
In this paper, we review recent computational techniques which make it possible to separate interannual climate variability of seasonal means into chaotic and potentially predictable components. Based on analysis of variance and frequency analysis of daily time series, the

*Bureau of Meteorology Research Centre, PO Box 1289K, Melbourne 3001, Australia. mailto:c.frederiksen@bom.gov.au

${ }^{\dagger}$ National Institute of Water and Atmospheric Research, PO Box 1491 Kilbirnie, Wellington, NEW ZEALAND

${ }^{0}$ See http: //anziamj . austms .org. au/V42/CTAC99/Fred for this article and ancillary services, (c) Austral. Mathematical Soc. 2000. Published 27 Nov 2000.
\end{abstract}


techniques are applicable to both observed data sets and ensembles of multidecadal simulations using atmospheric general circulation models forced by observed sea surface temperatures and different initial conditions. A new technique for validating the interannual variability in ensembles of model simulations is also outlined. The methodologies have been applied to a study of the interannual variability of the global $200 \mathrm{hPa}$ geopotential height field.

\section{Contents}

1 Introduction

C610

2 Methodology: Analysis of variance

C611

2.1 Model ... . . . . . . . . . . . . . C612

2.2 Observations. . . . . . . . . . . . . . . C615

2.3 Correlation and Model Validation . . . . . . . . . C616

3 Data

C619

4 Results

C620

5 Conclusions

C623

References

C625 


\section{Introduction}

Theoretically, dynamically extended seasonal forecasts, beyond the limit of predictability of individual synoptic weather systems (typically 10-15 days), are possible because atmospheric boundary forcings, such as, for example, sea surface temperature (SST) and vegetation cover, which evolve on slower time scales than that of the synoptic systems, largely determine the averaged weather features on a seasonal (1-3 month averages) time scale. However, even on this time scale and for seasonal averages, the climate system is still inherently chaotic [8] and it is of interest to determine how much of the climate variability, at different geographical locations, is in fact predictable.

A number of studies have considered the question of potential long-range predictability of climate variability. These have involved ensembles of multidecadal simulations using atmospheric general simulation models (AGCMs) with observed (SSTs) and different initial conditions [7, 8, e.g.]. With such an approach, it has been possible to separate interannual variability into chaotic components (due to the sensitivity to initial conditions) and a potentially predictable, or forced, component (based on the ensemble average). Thus, it is possible to identify areas where different climate variables are potentially predictable.

Recently, a new technique [8] for separating the interannual variance of modelled climate variables into a forced component, a low frequency internal source component and a weather noise component has been proposed. Similar 
techniques have been applied to observations [4, e.g.] but in this case it is not possible to separate directly the forced and the low frequency components. Thus, for observations, potential predictability has generally been defined in terms of the variability of this combined component.

How well models reproduce the interannual variability of the components of seasonal means is also a topic of great interest. Ideally, in assessing, or validating, a model's simulation of climate variability, the emphasis should be on the extent to which the forced component of variation is correctly simulated since this is potentially predictable. Zheng and Frederiksen [8] proposed an estimation procedure for the correlation between the forced components of simulated and observed seasonal means. Here, we review these techniques in some detail, and apply them to the modelled and observed global $200 \mathrm{hPa}$ geopotential height field.

The plan of this paper is as follows. In Section 2, we discuss the methodology, the observed and model data sets are described in Section 3, and the results are presented in Section 4. Our conclusions are summarised in Section 5.

\section{Methodology: Analysis of variance}




\subsection{Model}

Given an ensemble of $S$ AGCM simulations, it is possible to separate the variance of a climate variable into chaotic and potentially predictable components if we assume that a daily anomaly from an ensemble of AGCM simulations is represented by a simple linear statistical model as

$$
x_{s y t}=\mu+\beta_{y}+\delta_{s y}+\varepsilon_{\text {syt }} .
$$

Here, $s=1, \ldots, S$ is the simulation number; $y=1, \ldots, Y$ is the year; and $t=1, \ldots, T$ is the day in a season of length $T$ days; $\mu$ represents an overall mean taken over all $s, y$ and $t ; \beta_{y}$ is the deviation from $\mu$ due to external forcings such as, for example ssT-forcing, which may change from year to year; $\delta_{s y}$ is the low frequency component (interannual, decadal etc. ) in simulation $s$ due to internal sources and is assumed to be purely random and independent from one simulation to another; $\varepsilon_{\text {syt }}$ represents daily weather noise. The $\left\{\varepsilon_{s y t}, t=1, \ldots, T\right\}$ are assumed to represent a stationary normal stochastic process with mean zero and are independent and identically distributed with respect to $s$ and $y$.

As in [8] and [9], it is convenient to introduce the convention of using a "circle" as a subscript whenever an average is done over that index. Thus, for example, from Equation (1) a seasonal mean anomaly derived from daily values is written as

$$
x_{\text {syo }}=\mu+\beta_{y}+\delta_{s y}+\varepsilon_{\text {syo. }}
$$


Also, the symbol $\hat{V}$ will be used to denote the estimated variance of a variable or the estimated covariance of two variables. The aim is then to separate the variance of the climate seasonal mean anomaly into its constituent parts,

$$
\hat{V}\left(x_{\text {syo })}=\hat{V}\left(\beta_{y}\right)+\hat{V}\left(\delta_{s y}\right)+\hat{V}\left(\varepsilon_{\text {syo }}\right) .\right.
$$

Following [7], the following estimates are possible. The variance of the seasonal mean anomaly averaged over all simulations is simply,

$$
\hat{V}\left(x_{\text {oyo }}\right)=\frac{1}{Y-1} \sum_{y=1}^{Y}\left(x_{\text {oyo }}-x_{\text {ooo }}\right)^{2} .
$$

An estimate of the variance of the combined weather noise and low frequency internal components can be derived by looking at the spread among the ensemble members. Thus,

$$
\hat{V}\left(\delta_{\text {sy }}+\varepsilon_{\text {syo }}\right)=\frac{1}{Y(S-1)} \sum_{y=1}^{Y} \sum_{s=1}^{S}\left(x_{\text {syo }}-x_{\text {oyo }}\right)^{2} .
$$

Hence, assuming a normality condition on the internal components,

$$
\hat{V}\left(\delta_{\text {oy }}+\varepsilon_{\text {oyo }}\right)=\hat{V}\left(\delta_{\text {sy }}+\varepsilon_{\text {syo }}\right) / S .
$$

The variability of the forced component $\hat{V}\left(\beta_{y}\right)$ can then be estimated by using either the condition of [7], (which assumes that the forced components $\left\{\beta_{y}\right\}$ are statistically independent of each other and the internal components), or 
the method of [8] (which uses Kolmogorov's strong law of large numbers). Either way it follows that,

$$
\hat{V}\left(\beta_{y}\right)=\hat{V}\left(x_{\text {oyo }}\right)-\hat{V}\left(\delta_{\text {oy }}+\varepsilon_{\text {oyo }}\right)
$$

from which it follows further that the variability of the seasonal mean can be written as,

$$
\hat{V}\left(x_{\text {syo }}\right)=\hat{V}\left(\beta_{y}\right)+\hat{V}\left(\delta_{\text {sy }}+\varepsilon_{\text {syo }}\right) .
$$

The variance of the weather component of the seasonal mean can be estimated using the approach of [9], where the seasonal average of the daily values is thought of as one observation in a time series obtained by applying a $T$-day running mean filter to the original time series $\left\{\varepsilon_{\text {syt }}\right\}$. The variance of this new time series is given by the integral of its spectral density function, which can be approximated by its periodogram. Thus, it follows that,

$$
\hat{V}\left(\varepsilon_{\text {syo }}\right)=\frac{1}{S Y T^{2}} \sum_{s=1}^{S} \sum_{y=1}^{Y}\left|\sum_{t=1}^{T} x_{s y t} e^{i t 2 \pi / T}\right|^{2} .
$$

The use of $\left\{x_{\text {syt }}\right\}$ in Equation (9) is possible because $\left\{\beta_{y}\right\}$ and $\left\{\delta_{s y}\right\}$ do not involve daily values and hence their contribution to the sum over $t$ is identically zero. Thus, $\left\{\varepsilon_{\text {syt }}\right\}$ and $\left\{x_{\text {syt }}\right\}$ are interchangeable in Equation (9). Again, assuming normality, it follows that,

$$
\hat{V}\left(\varepsilon_{\text {oyo }}\right)=\hat{V}\left(\varepsilon_{\text {syo }}\right) / S,
$$


and the independence of the weather and low frequency components assures that,

$$
\hat{V}\left(\delta_{\text {oy }}\right)=\hat{V}\left(\delta_{\text {oy }}+\varepsilon_{\text {oyo }}\right)-\hat{V}\left(\varepsilon_{\text {oyo }}\right) .
$$

Hence, it follows that,

$$
\hat{V}\left(\delta_{s y}\right)=\hat{V}\left(\delta_{o y}\right) \times S .
$$

Using Equations (7-9), it follows further that the combined variance of the external forced and low frequency components can be expressed as,

$$
\hat{V}\left(\beta_{y}+\delta_{\text {sy }}\right)=\hat{V}\left(\beta_{y}\right)+\hat{V}\left(\delta_{\text {sy }}\right)=\hat{V}\left(x_{\text {oyo }}\right)-\hat{V}\left(\varepsilon_{\text {oyo }}\right) .
$$

Thus, the variance due to each of the three components, the total chaotic component, and the combined contribution from the external forcing and low frequency components can be estimated explicitly for the model.

\subsection{Observations}

For the observations similar formulas apply, except that it is not possible to separate forced and low frequency internal components as in Equation (13). If we represent daily observed anomalies by

$$
x_{y t}^{\prime}=\mu^{\prime}+\beta_{y}^{\prime}+\delta_{y}^{\prime}+\varepsilon_{y t}^{\prime} .
$$


then the following formulas apply for the observations,

$$
\begin{gathered}
\hat{V}\left(x_{y o}^{\prime}\right)=\frac{1}{Y-1} \sum_{y=1}^{Y}\left(x_{y o}^{\prime}-x_{o o}^{\prime}\right)^{2}, \\
\hat{V}\left(\varepsilon_{y o}^{\prime}\right)=\frac{1}{Y T^{2}} \sum_{y=1}^{Y}\left|\sum_{t=1}^{T} x_{y t}^{\prime} e^{i t 2 \pi / T}\right|^{2}, \\
\hat{V}\left(\beta_{y}^{\prime}+\delta_{y}^{\prime}\right)=\hat{V}\left(x_{y o}^{\prime}\right)-\hat{V}\left(\varepsilon_{y o}^{\prime}\right) .
\end{gathered}
$$

Thus, the observed interannual variance can be decomposed into,

$$
\hat{V}\left(x_{y o)}^{\prime}=\hat{V}\left(\beta_{y}^{\prime}+\delta_{y}^{\prime}\right)+\hat{V}\left(\varepsilon_{y o}^{\prime}\right) .\right.
$$

If one has access to a "good" model, then the first term on the right hand side can be further decomposed if one assumes (ad hoc) that,

$$
\frac{\hat{V}\left(\beta_{y}^{\prime}\right)}{\hat{V}\left(\beta_{y}^{\prime}+\delta_{y}^{\prime}\right)}=\frac{\hat{V}\left(\beta_{y}\right)}{\hat{V}\left(\beta_{y}+\delta_{s y}\right)} .
$$

\subsection{Correlation and Model Validation}

In most modelling studies involving an ensemble of runs, the correlation between simulated ensemble seasonal means and observed seasonal means is 
often used as an index of AGCM skill in simulating the SST-forced variability. This measure, in fact, represents the forecast (simulation) skill for an ensemble of finite size (or simulation number) given a perfect SST forecast. Low correlation could be due to the strongly chaotic nature of the climate system. Thus, in this context, it is not an optimal index of AGCM skill. The correlation between the forced component of simulated seasonal means and the observed seasonal means represents the forecast skill for infinite simulations, because the ensemble mean of simulated seasonal means converges towards the simulated forced component as the simulation number increases. The difference between the two correlation coefficients discussed here is a measure of the possible improvement of the real-world forecast skill by adding more simulations. A fairer test of the model would be to determine the extent to which the forced component of variability is correctly simulated.

Zheng and Frederiksen [8] show that the boundary forcing is the only possible source of the covariability between the simulated and observed seasonal mean climate variables. Thus it follows from the independence of the low frequency, weather noise and forced components, and using Kolmogorov's strong law of large numbers, that,

$$
\hat{V}\left(x_{\text {oyo }}, x_{y o}^{\prime}\right) \approx \hat{V}\left(\beta_{y}, x_{y o}^{\prime}\right) \approx \hat{V}\left(\beta_{y}, \beta_{y}^{\prime}+\delta_{y}^{\prime}\right) \approx \hat{V}\left(\beta_{y}, \beta_{y}^{\prime}\right)
$$

where,

$$
\hat{V}\left(x_{\text {oyo }}, x_{y o}^{\prime}\right)=\frac{1}{Y-1} \sum_{y=1}^{Y}\left(x_{\text {oyo }}-x_{\text {ooo }}\right)\left(x_{y o}^{\prime}-x_{o o}^{\prime}\right) .
$$


Hence, using the results of the two previous sections, it is easy to derive the following correlation estimates,

$$
\begin{gathered}
\hat{C}\left(x_{\text {oyo }}, x_{y o}^{\prime}\right)=\hat{V}\left(x_{\text {oyo }}, x_{y o}^{\prime}\right) /\left[\hat{V}\left(x_{\text {oyo }}\right) \hat{V}\left(x_{y o}^{\prime}\right)\right]^{\frac{1}{2}}, \\
\hat{C}\left(\beta_{y}, x_{y o}^{\prime}\right)=\hat{V}\left(\beta_{y}, x_{y o}^{\prime}\right) /\left[\hat{V}\left(\beta_{y}\right) \hat{V}\left(x_{y o}^{\prime}\right)\right]^{\frac{1}{2}}, \\
\hat{C}\left(\beta_{y}, \beta_{y}^{\prime}+\delta_{y}^{\prime}\right)=\hat{V}\left(\beta_{y}, \beta_{y}^{\prime}+\delta_{y}^{\prime}\right) /\left[\hat{V}\left(\beta_{y}\right) \hat{V}\left(\beta_{y}^{\prime}+\delta_{y}^{\prime}\right)\right]^{\frac{1}{2}}, \\
\hat{C}\left(\beta_{y}, \beta_{y}^{\prime}\right)=\hat{V}\left(\beta_{y}, \beta_{y}^{\prime}\right) /\left[\hat{V}\left(\beta_{y}\right) \hat{V}\left(\beta_{y}^{\prime}\right)\right]^{\frac{1}{2}} .
\end{gathered}
$$

The last equation is only possible if we assume Equation (19) is valid.

As mentioned above, a model's skill at reproducing observed climate variability has traditionally been measured by the correlation $\hat{C}\left(x_{\text {oyo }}, x_{\text {yo }}^{\prime}\right)$. Ideally, a better measure is $\hat{C}\left(\beta_{y}, \beta_{y}^{\prime}\right)$, but it can only be determined if we assume Equation (19). However, it is possible to put a lower bound on $\hat{C}\left(\beta_{y}, \beta_{y}^{\prime}\right)$, since it follows from Equations $(22-24)$ that, for positive $\hat{C}\left(x_{\text {oyo }}, x_{y o}^{\prime}\right)$,

$$
\hat{C}\left(x_{\text {oyo }}, x_{y o}^{\prime}\right) \leq \hat{C}\left(\beta_{y}, x_{y o}^{\prime}\right) \leq \hat{C}\left(\beta_{y}, \beta_{y}^{\prime}+\delta_{y}^{\prime}\right) \leq \hat{C}\left(\beta_{y}, \beta_{y}^{\prime}\right) .
$$




\section{Data}

The simulated data used in this study are the daily $200 \mathrm{hPa}$ geopotential heights from an ensemble of 4 runs of the Bureau of Meteorology Research Centre (BMRC) AGCM for the period March 1950-February 1990. The model has a horizontal truncation at rhomboidal wavenumber $31\left(2.5^{\circ}\right.$ lat., $3.75^{\circ}$ lon. grid) and has 9 vertical sigma levels. The model dynamics and physical parametrizations are detailed in [2] and [5] and references therein. The runs were forced by observed global sea surface temperature and sea ice taken form the Global Ice Sea Surface Temperature (GISST) dataset [6]. The carbon dioxide concentrations was fixed at $345 \mathrm{ppmv}$. Thus the effects of increasing carbon dioxide, and aerosol concentrations, have not been modelled. To ensure a smooth and balanced start, the initial atmospheric conditions were taken from a ten year AMIP (1979-1988) and a thirteen year (1950-1962) simulation of the model. Specifically, initial conditions were taken at $11 \mathrm{Z}$ on 1 and 2 December 1987, and 3 and 4 December 1962. The set of experiments performed is described more fully in [1].

We have taken as our "proxy" observed data the daily $200 \mathrm{hPa}$ geopotential height field from the National Centre for Environmental Prediction (NCEP) and National Centre for Atmospheric Research (NCAR) reanalyses [3], over the period 1958 through 1998. This data has been linearly interpolated onto the model grid. The analysis has been applied to both the modelled and the observed data for the common period 1958-1991 and to the SeptemberOctober-November (SON) seasonal mean. 


\section{Results}

As an example of the useful of the techniques discussed in Section 2, we have applied them to the $200 \mathrm{hPa}$ geopotential height field for son, using daily data from AGCM simulations and the NCEP/NCAR reanalysis for the period 1958-1991. The model, as is the case with many AGCMs, underestimates the total inter-annual variance of the height field in all seasons, especially in the middle to high latitudes (not shown). However, the spatial patterns of variation are similar and the fractions of the model variance explained by the weather noise component and the combined low frequency and forced components are similar to the observed. This can be seen from Figures 1 and 2 which show the contribution from each component expressed as a fraction of the total variance.

Figures 1 and 2 show that the weather noise and low frequency internal components have the greatest contribution in the extratropics of both hemispheres, and have only a minimal contribution in the tropics and subtropics. In contrast, this latter region is largely dominated by external forcing. The model weather noise component is similar to the observed, except that the main contribution in the Southern Hemisphere is displaced more equatorward. Similarly, the model seems to underestimate the influence of external forcing and low frequency dynamics in some extratropical regions, notably over North America and Greenland and in the southern parts of the Pacific and Indian Ocean. 

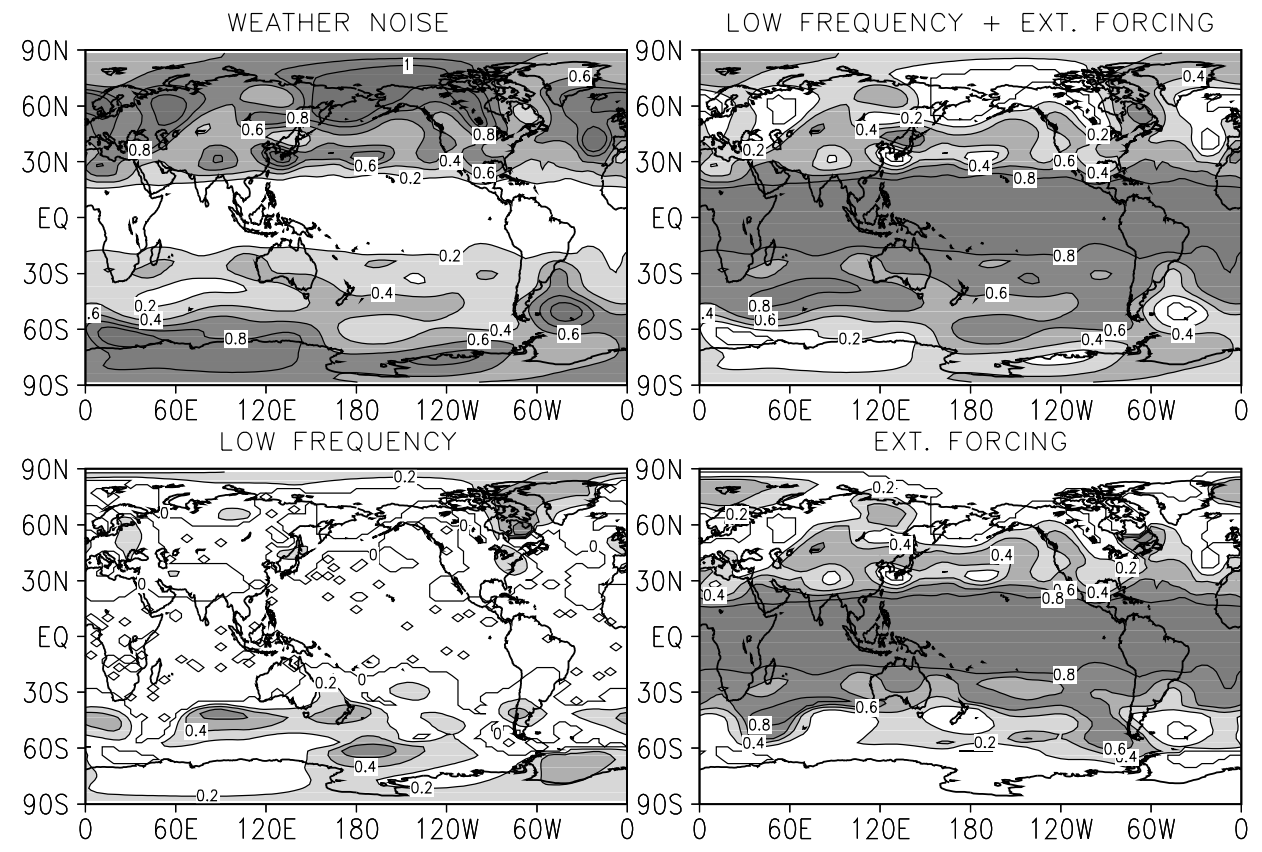
0
0.2
0.4
0.6
0.8
1

Figure 1: Components of variance of the observed $200 \mathrm{hPa}$ height expressed as a fraction of the total variance. 

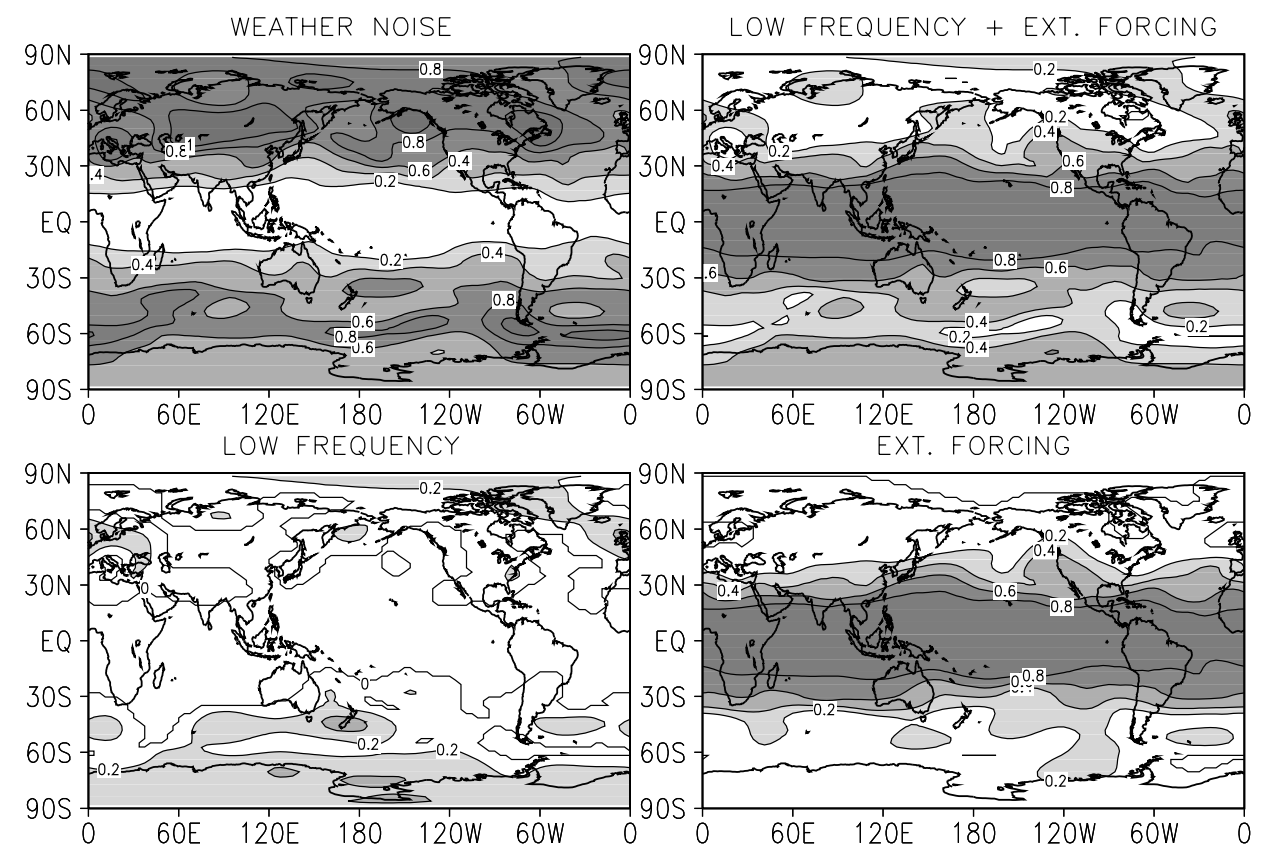
0
0.2
0.4
0.6
0.8
1

Figure 2: As in Figure 1 but for the model. 
Figure 3 shows the four possible correlation maps between model and observed. When the correlation between the total fields is negative in some geographical regions, these areas are left blank in the other correlation plots We can see that the correlation between model and observed increases as the chaotic components are successively removed. This is particularly true for the mid-latitudes. Also the difference between the correlation between the total fields and the correlation between the model forced and observed total field is not that much. This suggests that little extra skill would be obtained by taking a larger ensemble size during sON. This is not true in all seasons. For example, during December-January-February (not shown) much bigger differences are found suggesting a larger ensemble size would help.

\section{Conclusions}

We have discussed a number of techniques for separating the inter-annual variance of climate variables into a weather noise, low frequency internal and forced components. The latter represents the potentially predictable component. An estimation procedure for the correlation between the forced components of the simulated and observed seasonal means has also been proposed. This is a fairer measure of model skill than that currently used, that is, the correlation between the total fields.

The techniques have been applied to the $200 \mathrm{hPa}$ geopotential height field from an ensemble of AGCM simulations and compared with the ob- 

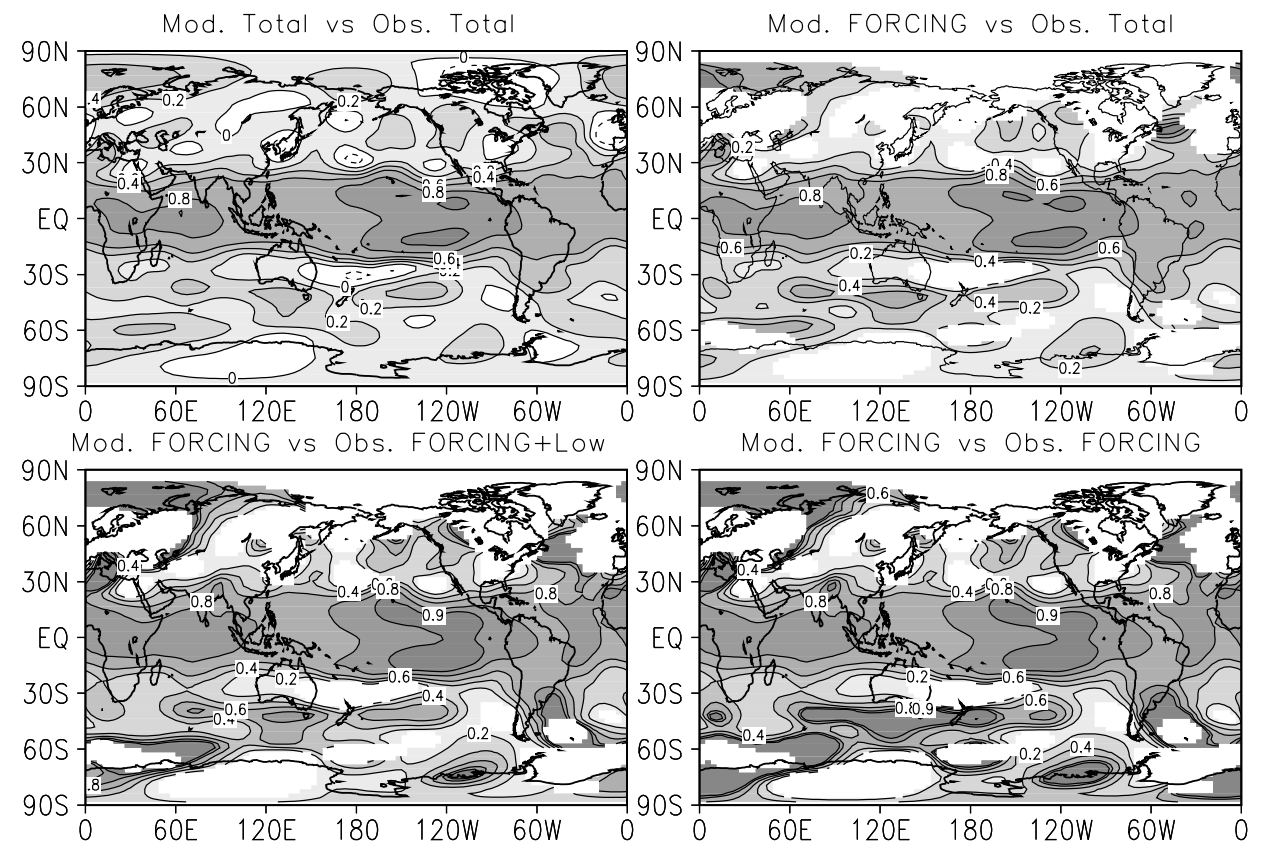

0
0.2
0.4
0.6

0.8

0.9

1

Figure 3: Correlations between modelled and observed components of variation. 
served (NCEP reanalysis). During SON, we found that the weather noise and low frequency internal components largely dominated the extratropics of the Northern Hemisphere and much of the Southern Hemisphere. Conversely, in the tropics/subtropics the external forcing largely dominated. Applying the correlation analysis, we found that, as expected, the correlation between model and observed increases as we progressively remove the chaotic components of variation. This was particularly true for the mid-latitudes. Largest correlations tended to occur in the 30S-30N latitude band where the model showed large potential predictability. This technique also allows us to see the impact of increasing the size of the ensemble size of simulations. We found for son that little extra would be gained by increasing the ensemble size from the four we used.

\section{References}

[1] C.S. Frederiksen, D. P. Rowell, R.C. Balgovind, and C. K. Folland. Multidecadal simulations of Australia rainfall variability: the role of SSTs. J. Climate, 12:357-379, 1999. C619

[2] T.L. Hart, W. Bourke, B.J. McAvaney, B.W. Forgan, and J.L. McGregor. Atmospheric general circulation simulations with the BMRC global spectral model: the impact of revised physical parameterizations. J. Climate, 3:436-459, 1990. C619 
[3] E. Kalnay, M. Kanamitsu, et al. The NCEP/NCAR 40-year reanalysis project. BAMS, 77:437-471, 1996. C619

[4] R.A. Madden. Estimates of the natural variability of time averaged sea level pressure. Mon. Wea. Rev., 104:942-952, 1976. C611

[5] B.J. McAvaney and R.A. Colman. The AMIP experiment: the BMRC AGCM Configuration. BMRC Report, 38, 43pp, 1993. C619

[6] D.E. Parker, P.D. Jones, C.K. Folland, and A. Bevan. Interdecadal changes of surface temperature since the late nineteenth century. J. Geophys. Res., 99:14373-14399, 1994. C619

[7] D.P. Rowell. Assessing potential predictability with an ensemble of multi-decadal GCM simulations. J. Climate, 11:109-120, 1998. C610, C613, C613

[8] X. Zheng and C.S. Frederiksen. Validating interannual variability in an ensemble of AGCM simulations. J. Climate, 12:2386-2396, 1999. C610, C610, C610, C611, C612, C614, C617

[9] F.W. Zwiers. A potential predictability study conducted with an atmospheric general circulation model. Mon. Wea. Rev., 115:2957-2974, 1987. C612, C614 